\title{
Treatment Updates in Acute Myeloid Leukemia
}

\author{
Presented by Alexander E. Perl, MD, MS
}

\begin{abstract}
The NCCN Guidelines for the management of acute myeloid leukemia (AML) have, for the most part, remained unchanged from 2020, with some shifts in emphasis, fine-tuning, and several important updates in the 2021 version. The guidelines now emphasize physiologic age rather than chronologic age, and recommend that fit patients should receive intensive chemotherapy. Actionable genetics are being used to guide selection of therapy. Minimal residual disease assessment after induction therapy is now incorporated in the guidelines. New therapeutic options include oral azacitidine and combination venetoclax + azacitidine, and preliminary evidence suggests that IDH inhibitors will come to the fore in the treatment of AML.

J Natl Compr Canc Netw 2021;19(5.5):652-654
\end{abstract} doi: $10.6004 /$ jnccn.2021.5008

"In the past few years, the NCCN Clinical Practice Guidelines in Oncology [NCCN Guidelines] for Acute Myeloid Leukemia [AML] have had some changes due to new drug approvals. This year, the updates are more incremental than revolutionary, but there are some important updates," stated Alexander E. Perl, MD, MS, Associate Professor of Medicine, Abramson Cancer Center at the University of Pennsylvania, during the NCCN 2021 Virtual Annual Conference. "For one thing, the current guidelines emphasize assessment according to physiologic age rather than chronologic age, whereas older guidelines emphasized chronologic age," he continued. "Now we look at fitness to make therapeutic choices."

"Within any age category, physiologic age, or fitness level, genetics are increasingly being used to drive therapy selection. In the past, we used genetics largely to track patients for persistence of disease, but what is new and different and has become standard is that we are using mutations to drive therapy choices," he said. Mutations are also being used to track the level of disease burden, and minimal residual disease (MRD)—which is standard in acute lymphoblastic leukemia-is increasingly used in AML and starting to become incorporated into the guidelines. The strongest evidence for MRD clearance guiding therapy is in core binding factor (CBF) AML, but increasingly, detection of NPMI can also be used to make treatment decisions.

The most reliable method for detecting gene fusions is RT-PCR, and there is evidence supporting the use of RT-PCR for CBF gene rearrangements in treatment decisions. For patients with non-CBF AML, Dr. Perl said there are no standardized assays for MRD assessment, but flow cytometry is used, which he said is "operatordependent."

\section{Acute Promyelocytic Leukemia}

"Acute promyelocytic leukemia [APL] is the most important subgroup in AML, because it is the most curable. We finally have long-term follow-up data from the GIMEMAled APL 0406 study showing that all-trans retinoic acid (ATRA) and arsenic as frontline therapy leads to superb cure rates in low-risk APL [ie, WBC counts $<10,000 / \mu \mathrm{L}]$," Dr. Perl noted. ${ }^{1}$ The survival benefit was evident with 5year follow-up, and the regimen appears to avoid serious late-onset adverse events such as late relapses and secondary AML, he continued.

"The value of monitoring postinduction recovery marrow biopsy is modest in patients with low-risk APL (ie, initial WBC count $<10,000 / \mu \mathrm{L}$ ). RT-PCR PML-RARA transcript monitoring for 2 years after completion of therapy," Dr. Perl said. "Cure rates remain excellent, and late events are rare."

\section{Non-APL AML}

Although novel agents are highly active in AML, fit older patients benefit from intensive chemotherapy, and it can lead to cure. "Although the cure rate is not as high in older patients, some patients who receive chemotherapy alone or chemotherapy plus transplant and achieve remission never experience relapse," Dr. Perl stated.

Risk stratification has remained largely unchanged in the 2021 NCCN Guidelines. The guidelines now recommend MRD testing over KIT mutation status to determine risk level among patients with CBF AML. "From the 
get-go, fit patients with non-APL AML need a comprehensive genetic assessment. We generally recommend that induction therapy be withheld for a few days if patients are clinically stable until a genetic assessment can help determine the best therapy. The genetics of AML drive the approach to induction therapy, such as whether to use $7+3$ alone, add a third drug, or use liposomal cytarabine and daunorubicin," Dr. Perl said.

For asymptomatic patients, it is important to wait for the results of a genetic assessment before beginning treatment. Subsets of patients benefit from novel induction strategies, such as gemtuzumab ozogamicin (GO) added to induction and consolidation therapy for those with $\mathrm{CBF}$ leukemias, liposomal formulations for older patients with secondary leukemias, and the addition of midostaurin for those with FLT3 mutations. "It is safe to withhold intensive therapy for up to 2 weeks as long as the patient is clinically stable and not highly symptomatic. I would not recommend waiting to treat for rapidly progressing, highly symptomatic disease. The gains from intensive therapy are significant, and you don't want to lose a window of opportunity," he explained.

\section{CBF Leukemia}

Recommendations for induction therapy remain unchanged for 2021 in CBF leukemia. GO improves survival in this group of patients and the benefit extends to those with FLT3 mutation-positive disease ${ }^{2}$; approximately $15 \%$ of patients with CBF disease have FLT3 mutations. There is a risk for hepatotoxicity, and therefore lower doses are used, and it takes approximately 90 days for the drug to clear before patients can go on to transplant.

Risk stratification has changed somewhat in the updated guidelines. Although KIT mutation assessment is included, MRD is a stronger predictor of relapse for patients in remission. "Patients who fail to clear $t(8 ; 21)$ or inv(16) transcript are not guaranteed to relapse, but they are at higher risk. If there is persistence of MRD by RTPCR, it may be prudent to perform a transplant workup at first remission, even if the patient has CBF AML," Dr. Perl noted.

Midostaurin is approved for FLT3-mutated AML. New data on European LeukemiaNet (ELN) genetic groups show that favorable, intermediate, and adverse risk groups and all cytogenetic subtypes had a survival benefit from midostaurin versus placebo. ${ }^{3-5}$ Currently, the induction strategy with midostaurin is recommended only for intermediate-risk karyotype, but in actuality it isn't clear whether midostaurin or GO is preferable in favorable-risk patients. "Most people recommend GO in patients with both mutations," Dr. Perl said. He thinks midostaurin may also be of benefit for adverse-risk patients based on recently published data. ${ }^{4}$

\section{Postremission Therapy and Transplant Indications}

In the updated version of the NCCN Guidelines, postremission therapy and transplant indications are largely the same. Patients who are candidates for intensive chemotherapy and transplant are recommended to receive that treatment. "If a patient can be treated intensively, they should be, because it can cure them," Dr. Perl stated. "Oral azacitidine seems to benefit patients who are eligible for intensive chemotherapy but don't complete consolidation and can't go on to transplant," he added. Oral azacitidine was approved in 2020 for the postremission setting, although he pointed out that it was only tested in patients who had undergone intensive induction therapy. "Oral azacitidine was not designed to replace intensive therapy/ hematopoietic stem cell transplant post-remission," he added.

He noted that oral azacitidine (CC486) is not bioequivalent to the subcutaneous and intravenous formulations of azacitidine, and is given at a different dose and duration. The recommended dose and schedule is $300 \mathrm{mg}$ once daily for days 1 to 14 , and repeated every 28 days.

The international, randomized, placebo-controlled, double-blind QUAZAR study enrolled 472 patients from 148 sites in 23 countries. ${ }^{6}$ Eligible patients were in first remission on intensive induction \pm consolidation therapy, were aged $>55$ years (median, 68 years), had intermediateor poor-risk cytogenetics, were not $\mathrm{CBF}$ rearranged, and were transplant-ineligible. Study participants were randomized to oral azacitidine versus placebo and were followed until death. At a median follow-up of 41.2 months, overall survival was substantially improved by oral azacitidine (median, 24.7 vs 8.9 months for placebo; $P=.0009$ ).

"Ultimately, the survival curves come together, so it is not clear if we are curing those patients," Dr. Perl noted. "The primary benefit in patients with or without prior consolidation therapy is prevention of relapse." A subgroup analysis found that the benefit of oral azacitidine extended to every subgroup, including younger or older age, MRD-positive or MRD-negative disease, and intermediate- or poor-risk cytogenetics. ${ }^{6}$

Every drug has adverse effects, and oral azacitidine carries the risk of significant gastrointestinal toxicity, for which preemptive antiemetics are recommended. Neutropenia is common, but can be managed with dose reduction from 300 to $200 \mathrm{mg}$. Febrile neutropenia is managed with antibiotics. There were no treatmentrelated deaths in the QUAZAR study. ${ }^{6}$

The NCCN Guidelines do not have data on the use of FLT3 inhibitors posttransplant. Two randomized trials support posttransplant sorafenib in patients with FLT3ITD mutation, ${ }^{7,8}$ and a phase III trial is evaluating gilteritinib as maintenance therapy posttransplant 
(ClinicalTrials.gov identifier: NCT02997202). “These studies will show if patients treated with modern therapies benefit from posttransplant maintenance therapy. It is not clear if this will make it into the NCCN Guidelines. Stay tuned," Dr. Perl told listeners.

\section{Venetoclax + Parenteral Azacitidine}

Venetoclax + parenteral azacitidine represents a new standard of care in 2021 for patients who are unfit for intensive chemotherapy. "This is a major update that changes how we approach older patients or those who are unfit for intensive chemotherapy," Dr. Perl stated.

The phase III placebo-controlled VIALE-A trial enrolled patients with newly diagnosed AML ineligible for induction therapy due to comorbidity and those aged $\geq 75$ years. ${ }^{9} \mathrm{CBF}$ leukemia was excluded, in addition to a prior hypomethylating agent or prior venetoclax. Median overall survival was 14.7 months for the combination versus 9.6 for azacitidine + placebo $(P<.001)$. Venetoclax + azacitidine improved complete response rates and overall survival across many subcategories of AML, including both intermediate and adverse risk karyotype, de novo and secondary AML, and patients with genetic mutations in IDH, FLT-3, NPM1, or TP53. "The clearest benefit was for patients with $I D H$ mutations, but there were gains across the board for every genetic subtype," Dr. Perl noted. "Median survival of approximately 15 months is quite impressive for this patient population."

Dr. Perl provided tips for using venetoclax + azacitidine that are spelled out in the NCCN Guidelines. Both drugs should be started together, and patients should be cytoreduced to $<25,000 / \mu \mathrm{L}$ WBC before treatment initiation. Monitoring for tumor lysis syndrome should be performed during the 3 days of venetoclax ramp-up. If patients are taking concurrent moderate or strong CYP3A4 inhibitors, the dose of venetoclax should be adjusted downward. Response should be checked at the end of cycle 1 .

"It is important to monitor for response early, because typically response is seen after 1 or 2 cycles," he said. If counts are still recovering, initiation of the second cycle should be delayed for 1 to 2 weeks. Granulocyte colony-stimulating factor can be used in patients responding to treatment, particularly in subsequent cycles.

Treatment-emergent cytopenias in patients experiencing response may warrant dosing adjustment on subsequent cycles. Clinicians should consider marrow biopsy every 3 to 6 months to confirm ongoing response. The benefits of venetoclax/azacitidine in patients previously treated with hypomethylating agents are uncertain; this has not been studied.

\section{Ivosidenib}

Looking toward the future, "intriguing" single-arm studies of frontline IDH inhibitors suggest ivosidenib may find a role in the initial treatment of AML. "High complete response rates and promising survival have been shown with or without additional agents. The data are interesting, but for most patients, venetoclax/azacitidine may be preferable as initial therapy and ivosidenib combinations are not yet FDA-approved," Dr. Perl said.

Disclosures: Dr. Perl has disclosed receiving honoraria from and serving as a scientific advisor for AbbVie, Inc., Actinium Pharmaceuticals, Astellas Pharma US, Inc., Celgene Corporation, Daiichi-ankyo Co., Genentech, Inc., Loxo Oncology, Inc., Sumitomo Dainippon, and Syndax; receiving grant/ research support from AbbVie, Inc., Astellas Pharma US, Inc., Daiichi-ankyo Co., and Fujifilm Corporation; and receiving consulting fees from AbbVie, Inc., Astellas Pharma US, Inc., OncoNova, BeatAML LLC, Daiichi-ankyo Co. and Forma Therapeutics.

Correspondence: Alexander E. Perl, MD, MS, Abramson Cancer Center at the University of Pennsylvania, 3400 Civic Center Boulevard, Perelman 12 South, Philadelphia, PA 19104. Email: alexander.perl@pennmedicine.upenn.edu

\section{References}

1. Cicconi L, Platzbecker $U$, Avvisati $G$, et al. Long-term results of all-trans retinoic acid and arsenic trioxide in non-high-risk acute promyelocytic leukemia: update of the APL0406 Italian-German randomized trial. Leukemia 2020;34:914-918.

2. Hills RK, Castaigne S, Appelbaum FR, et al. Addition of gemtuzumab ozogamicin to induction chemotherapy in adult patients with acute myeloid leukemia: a meta-analysis of individual patient data from randomized controlled trials. Lancet Oncol 2014;15: 986-989.

3. Stone RM, Mandrekar S, Sanford BL, et al. Midostaurin plus chemotherapy for acute myeloid leukemia with a FLT3 mutation. N Engl J Med 2017;377:454-464.

4. Döhner K, Thiede C, Jahn N, et al. Impact of NPM1/FLT3-ITD genotypes defined by the 2017 European LeukemiaNet in patients with acute myeloid leukemia. Blood 2020;135:371-380.

5. Voso MT, Larson RA, Jones D, et al. Midostaurin in patients with acute myeloid leukemia and FLT3-TKD mutations: a subanalysis from the RATIFY trial. Blood Adv 2020;4:4945-4954.

6. Wei AH, Döhner H, Pocock $C$, et al. Oral azacitidine maintenance therapy for acute myeloid leukemia in first remission. N Engl J Med 2020;383:2526-2537.

7. Burchert A, Bug G, Fritz LV, et al. Sorafenib maintenance after allogeneic hematopoietic stem cell transplant for acute myeloid leukemia with FLT3-internal tandem duplication mutation (SORMAIN). J Clin Oncol 2020;38:2993-3002.

8. Xuan L, Wang $Y$, Huang $F$, et al. Sorafenib maintenance in patients with FLT3-ITD acute myeloid leukemia undergoing allogeneic hematopoietic stem-cell transplantation: an open-label, multicenter, randomized phase 3 trial. Lancet Oncol 2020;21:1201-1212.

9. DiNardo $C D$, Jonas BA, Pullarkat $V$, et al. Azacitidine and venetoclax for previously untreated acute myeloid leukemia. N Engl J Med 2020;383:617-629. 